\title{
Comparison of Clinical Results and Risk of Patellar Injury between Attune and PFC Sigma Knee Systems
}

\author{
Sang Jun Song, $\mathrm{MD}, \mathrm{PhD}^{1}$, Se Gu Kang, $\mathrm{MD}^{1}$, Cheol Hee Park, $\mathrm{MD}^{2}$, and Dae Kyung Bae, $\mathrm{MD}$, PhD \\ ${ }^{1}$ Department of Orthopaedic Surgery, College of Medicine, Kyung Hee University, Seoul; ${ }^{2}$ Department of Medicine, Graduate School, Kyung Hee University, Seoul; \\ ${ }^{3}$ Department of Orthopaedic Surgery, Seoul Sacred Heart General Hospital, Seoul, Korea
}

Purpose: The purposes of this study were to compare clinical results after total knee arthroplasty (TKA) using the Attune and PFC Sigma knee designs and to investigate whether the use of the Attune prosthesis increased the risk of patellar injury in Asian patients.

Materials and Methods: Three hundred knees that underwent TKA using Attune (group A) were compared to 300 knees that underwent TKA using PFC Sigma (group B). The Knee Society Knee Score (KS) and Function Score (FS), and range of motion (ROM) were compared. The residual patellar thickness was compared to evaluate the risk of patellar injury.

Results: The postoperative KS and ROM of group A were better than those of group B (93.1 vs. 88.8, p $<0.001$ and $131.4^{\circ}$ vs. $129.0^{\circ}$, p=0.008, respectively). The postoperative FS did not differ significantly between the two groups ( 80.9 vs. $78.7, \mathrm{p}=0.427)$. The residual patella was thinner in group A (14.8 mm vs. $15.7 \mathrm{~mm}, \mathrm{p}=0.003)$, which made up a higher proportion of the high-risk group for patellar fractures with a residual thickness of $<12 \mathrm{~mm}(7.5 \%$ vs. $2.1 \%$, $\mathrm{p}=0.003)$.

Conclusions: TKA using the Attune prosthesis provided more favorable clinical results than TKA using PFC Sigma. However, the risk of injury in the residual patella was increased with use of the Attune prosthesis in Asian patients.

Keywords: Knee, Arthroplasty, Patella, Injury, Risk

\section{Introduction}

Any problems involving the patella after total knee arthroplasty (TKA) can influence overall knee function ${ }^{1)}$. In particular, periprosthetic patellar fractures, albeit infrequent, have been considered a troublesome complication because return to prefracture function is rare even with anatomic reduction, healing, and reconstitution of the extensor mechanism ${ }^{1-3)}$. It is known that the thickness of the residual patella is one of the most important factors affecting the risk of periprosthetic patellar fractures. Many

Received March 7, 2018; Revised April 24, 2018;

Accepted May 27, 2018

Correspondence to: Cheol Hee Park, MD

Department of Medicine, Graduate School, Kyung Hee University, 23

Kyungheedae-ro, Dongdaemun-gu, Seoul 02447, Korea

Tel: +82-2-958-9489, Fax: +82-2-964-3865

E-mail: rdohead@hanmail.net

This is an Open Access article distributed under the terms of the Creative Commons Attribution Non-Commercial License (http://creativecommons.org/licenses/by-nc/4.0/) which permits unrestricted non-commercial use, distribution, and reproduction in any medium, provided the original work is properly cited. previous studies have proposed that if the resurfaced patella is too thin, there may be an increased risk of fracture due to increased stress on the residual native bone $e^{4-6)}$.

Many manufacturers have modified their TKA prosthesis designs to improve functional outcomes. The Attune prosthesis (Depuy Synthes, Warsaw, IN, USA) is a modified version of the PFC Sigma (Depuy Synthes). The theoretical advantages of the Attune prosthesis are increased conformity between the femoral component and polyethylene insert, an extensive range of sizes for diverse populations, optimization of patellofemoral conformity, and an improved polyethylene insert locking mechanism $(\text { Fig. } 1)^{7-10)}$. These design modifications resulted in improvement in patellofemoral clinical outcomes, decreasing the incidence of anterior knee pain and patellar crepitus ${ }^{8-10)}$.

However, despite such advantages, there are some design features of the current prosthesis that might cause problems. The thickness of the Attune patellar component is greater than that of the PFC Sigma. The thicknesses of the Attune patellar components are $8.5,9.0,9.5,10$, and $10.5 \mathrm{~mm}$ for sizes $29,32,35,38$, and 41 , respectively ${ }^{7)}$. By contrast, the thicknesses of the PFC Sigma 
A

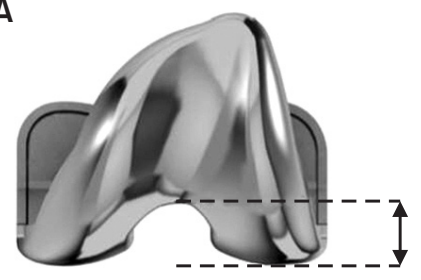

Attune

B
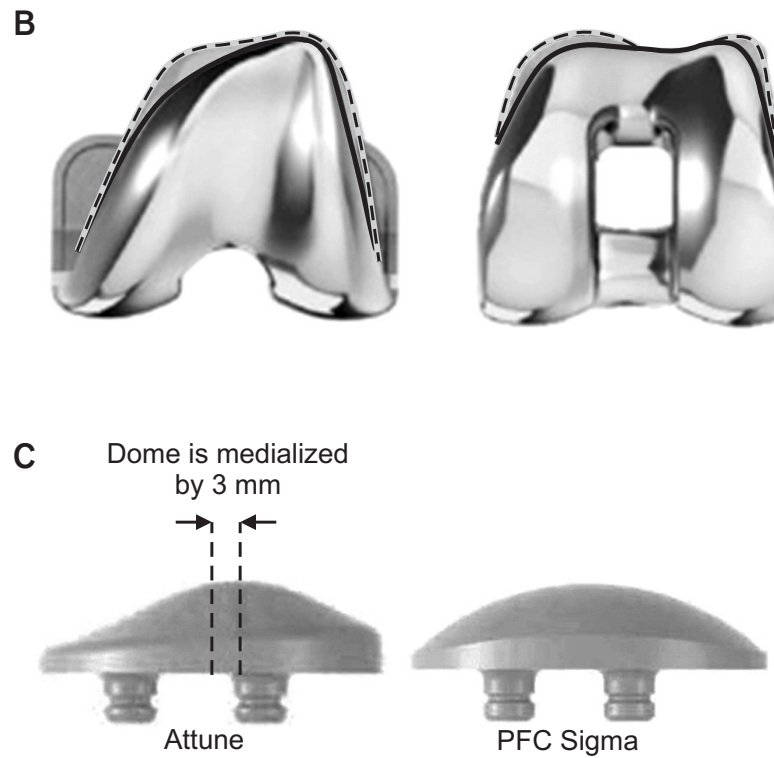

Fig. 1. Modified design features of Attune compared to PFC Sigma. (A) The trochlear groove of Attune is extended more distally than PFC Sigma, resulting in a reduced intercondylar box ratio. (B) Reduced width and thickness of Attune (inner dimension; solid line) compared to PFC Sigma (outer dimension; dotted line). (C) The Attune prosthesis has a medialized dome patellar component for optimizing patellofemoral conformity.

patellar components are $8.0 \mathrm{~mm}$ for size $29 ; 8.5 \mathrm{~mm}$ for sizes 32 , 35 , and 38; and $11 \mathrm{~mm}$ for size $41^{11)}$. Thus, considering that the postoperative patellar-patellar component composite thickness should be similar with the thickness of the original patella, the residual bone stock should be shallow in the knees with the current prosthesis, which increases the possibility of patellar fractures especially in patients with a small patella ${ }^{12,13)}$. In general, Asians have a smaller knee with a thinner patella compared to Caucasian; therefore, the risk of patellar injury might be increased when the Attune prosthesis is used in Asian patients ${ }^{12)}$.

The purposes of this study were to compare clinical and radiographic results after TKA using the Attune and PFC Sigma knee systems and to investigate whether the use of Attune prosthesis increased injury risk to the patella in Asian patients. It was hypothesized that the clinical results of Attune prosthesis would be comparable to or better than those of the PFC Sigma prosthesis and that the injury risk to the patella could be increased with use of Attune.

\section{Materials and Methods}

\section{Patients}

All consecutive patients who underwent TKA using Attune between November 2014 and June 2015 (group A) were enrolled in this study. During this period, 300 TKAs were performed with this prosthesis in 273 patients. For each patient reviewed, we matched a control from our patient database who had undergone primary TKA with PFC Sigma (group B) with respect to age, gender, body mass index, diagnosis, preoperative range of motion (ROM), and severity of preoperative deformity. The inclusion criterion was TKA performed by two senior surgeons with more than 20 years of experience in primary posterior stabilized TKA using the two prostheses. All patients underwent patella resurfacing due to severe patellofemoral arthritis. We excluded (1) patients with previous infection, fracture, or dislocation of the knee; (2) patients with previous high tibial osteotomy or revision TKA; and (3) patients with extra-articular deformity or severe bone loss. These patients were excluded because of the concern for anatomical distortion of the patella. The preoperative demographic data did not differ significantly between the two groups except the follow-up period (Table 1). The follow-up period was inevitably longer in group B because we have mainly used the Attune prosthesis instead of its predecessor (PFC Sigma) after introduction of the latest model. This study was approved by the Institutional Review Board (KHUH 2017-05-062). Informed consent was obtained from all patients before commencing the database review.

\section{Surgical Technique and Rehabilitation}

The medial parapatellar approach was used with a midline skin incision. All TKAs were planned to use a posterior stabilized prosthesis with patella resurfacing. Femoral and tibial bone resections were made with a modified measured resection technique. The transepicondylar axis was used as a reference for femoral component rotation. The tibial resection was set to be $0^{\circ}-3^{\circ}$ of the posterior slope in the sagittal plane. The reference line for tibial rotation was accurately aimed to pass through the medial third of the tibial tubercle and the second metatarsal bone or the middle of the talus. All osteophytes were removed. Any contracted medial or lateral soft tissue was carefully evaluated and selectively released where required.

To prepare the patella, the patella was resected with a sharp 
Table 1. Comparison of Patient Demographics between Groups

\begin{tabular}{lccc}
\hline \multicolumn{1}{c}{ Variable } & Group A $^{\mathrm{a})}$ & Group B $^{\mathrm{b})}$ & $\mathrm{p}$-value \\
\hline Operating period & Nov 2014-Jun 2015 & Jan 2013-Nov 2014 & $300(282)$ \\
No. of knees (patients) & $300(273)$ & $68.9 \pm 6.9$ & 0.102 \\
Age (yr) & $69.7 \pm 7.7$ & $290 / 10$ & 0.524 \\
Sex (female/male) & $287 / 13$ & $154 / 146$ & 0.806 \\
Right/left & $151 / 149$ & $26.1 \pm 3.3$ & 0.565 \\
Body mass index $\left(\mathrm{kg} / \mathrm{m}^{2}\right)$ & $26.5 \pm 3.6$ & $290 / 6 / 4$ & 0.455 \\
OA/RA/Others & $289 / 5 / 6$ & $120.2 \pm 20.7$ & 0.834 \\
Range of motion $\left({ }^{\circ}\right)$ & $119.8 \pm 25.7$ & Varus $11.7 \pm 7.5$ & 0.751 \\
Preoperative mechanical axis $\left(^{\circ}\right)$ & Varus $11.6 \pm 6.6$ & $33.3 \pm 9.0(21-76)$ & $<0.001$ \\
Follow-up period $($ mo $)$ & $24.8 \pm 6.0(16-37)$ & &
\end{tabular}

Values are presented as mean \pm standard deviation (range).

OA: osteoarthritis, RA: rheumatoid arthritis, Others: post-traumatic arthritis, infection sequelae, and hemophilic arthritis.

${ }^{a)}$ Group A patients who received the Attune prosthesis.

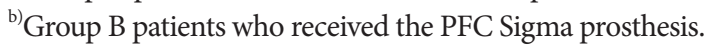

electric saw. For the evaluation of the even cut surface, the patella was divided into four quadrants and thickness was measured at the center of each quadrant using a caliper. The original patellar thickness was preserved or slightly decreased by about $0.5 \mathrm{~mm}$ with beveling of the uncovered lateral cut surface. The medialized dome patellar component was used for group A, and the oval dome patellar component was used for group B. Patellofemoral articulation was carefully evaluated with the no thumb technique. Lateral retinacular release was not performed because there was no case with patellar maltracking under tourniquet inflation.

The postoperative rehabilitation protocol was similar between the two groups ${ }^{14)}$. Isometric exercises using the extensor and flexor muscles were initiated shortly after operation. Drain was removed on the second postoperative day, followed by the initiation of active and assisted ROM exercises. Full weight bearing ambulation was started 4 days postoperatively to the extent that the patient's condition permitted.

\section{Clinical and Radiographic Evaluation}

The clinical scores recorded in our database were retrospectively analyzed. For the clinical evaluation, Knee Society Knee Score (KS) and Function Score (FS) were used to access pain and function $^{15)}$. The ROM was measured using a long-armed goniometer. Follow-up was conducted regularly at postoperative 3 months, 6 months, 1 year, and annually thereafter.

Pre- and postoperative anteroposterior (AP) and lateral radiographs and orthoroentgenograms (full-length standing AP radiographs) were used to assess limb alignment and component positioning. Pre- and postoperative mechanical axis was defined
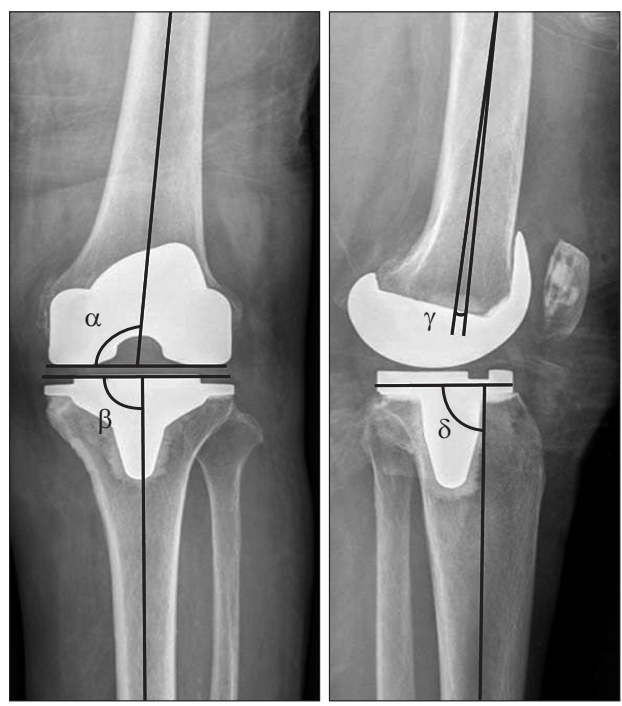

Fig. 2. Component positions according to the Knee Society radiological evaluation method.

as the angle between the femoral and tibial mechanical axes on orthoroentgenograms. Detailed analyses of the AP and lateral radiographs were conducted to evaluate the position of components with $\alpha, \beta, \gamma$, and $\delta$ angles using the Knee Society radiological evaluation method (Fig. 2) ${ }^{16}$.

The pre- and postoperative patella thickness and postoperative thickness of the residual patella were measured in the Merchant view of the knee ${ }^{17)}$. Patellar thickness was defined as the length of the thickest portion of the preoperative patella or postoperative prosthesis-patellar composite. The thickness of the residual patella was measured at the thickest portion of the remaining pa- 
tella after resurfacing (Fig. 3). Because a patella fracture is more likely to develop when the residual thickness is less than $12 \mathrm{~mm}$, patients with such thicknesses were categorized into a high-risk group for patellar fractures ${ }^{18,19)}$.

The quality of radiographic evaluation could be improved by standardization of the radiographic protocol with respect to the position of the knee and the distance between the X-ray beam and the cassette. The AP radiographs and orthoroentgenograms were taken with the patient standing with the knee fully extended and the feet slightly internally rotated to ensure forward placement of all anatomic landmarks ${ }^{20)}$. For the lateral radiographs, the knee was positioned in the same manner except the $\mathrm{x}$-ray beam was directed laterally at $90^{\circ}$ to the AP plane ${ }^{21)}$. The distance between the radiographic source and the patient's bone was 245 $\mathrm{cm}$, and that between the radiographic source and the cassette film was $260 \mathrm{~cm}^{22)}$.

When taking the Merchant radiographs, a standardized protocol was also used to minimize variances in the quality of radiographs. A leg position device equipped with an X-ray cassette holding slot was used to maintain uniformity in the angle and distances between the X-ray tube, the patella, and the cassette. The patient lay supine on the table with the knee flexed and supported at $45^{\circ}$ angle. The $\mathrm{x}$-ray beam was directed toward the feet at $30^{\circ}$ from the horizontal, and a film cassette set was positioned $30 \mathrm{~cm}$ below the knee. The angle and distance between the X-ray source, the patella, and the cassette were kept unchanged.

The images were transferred digitally to a picture archiving and communication system (PACS; Infinitt, Seoul, Korea). Assessment was performed on a 61-cm (24-inch) monitor (SyncMaster 2494HMN; Samsung, Seoul, Korea) in portrait mode using the PACS software. The minimum differences that the software could detect were $0.1^{\circ}$ in angle and $0.1 \mathrm{~mm}$ in length ${ }^{23)}$.

The radiographic evaluation of the Merchant view was per- formed on the PACS image setting the PACS ruler to be $10 \mathrm{~cm}$. Magnification ratio was evaluated through a pilot test using 30 knees, and it was found to be approximately 1.1 in the PACS image. The measurements on the Merchant view were adjusted taking into account the magnification ratio.

To minimize any observation bias, two independent investigators repeatedly performed all of the radiographic measurements with an interval of 2 weeks. The intra- and interobserver reliabilities of all measurements were assessed using the intraclass correlation coefficient, all values of which were greater than 0.8 . Thus, the radiographic measurements taken by one investigator who had more clinical experience than the other investigator were used in the analyses.

The actual occurrences of complications associated with the injury of patella, including periprosthetic fracture and loosening of the patellar component, were investigated during the follow-up period.

\section{Statistical Analysis}

Clinical and radiographic results were compared between groups A and B (independent Student $t$-test). The preoperative and postoperative clinical and radiographic results were also compared (paired $t$-test). The proportion of patients in the highrisk group for patella fracture was compared between the two groups (chi-square test).

To determine whether our sample had sufficient power to detect significant differences, we performed post-hoc power analyses using the significance levels set at an alpha of 0.05. A power $>80 \%$ was considered sufficient, and all of the variables that were significantly different met the criterion. Thus, we determined that our study was adequately powered.

Statistical analyses were performed using SPSS ver. 18.0 (SPSS Inc., Chicago, IL, USA), and a $\mathrm{p}<0.05$ was considered statistically significant.
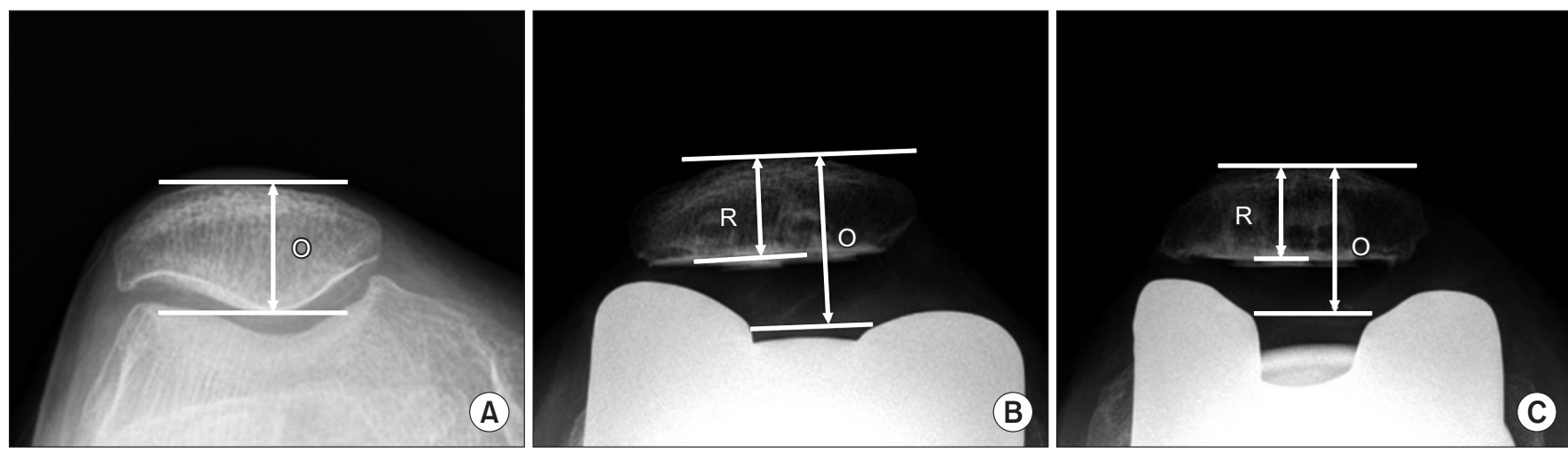

Fig. 3. Radiographic measurement of the thickness of the original and residual patella. (A) Original patella. (B) Attune. (C) PFC Sigma. O: original patellar thickness, R: residual patellar thickness. 
Table 2. Comparison of the Clinical Results between the Groups

\begin{tabular}{lccc}
\hline \multicolumn{1}{c}{ Variable } & Group A $^{\mathrm{a})}$ & Group B $^{\mathrm{b})}$ & $\mathrm{p}$-value \\
\hline Knee score & & & \\
Preoperative & $42.2 \pm 16.9$ & $43.9 \pm 9.0$ & 0.145 \\
Postoperative & $93.1 \pm 6.4$ & $88.8 \pm 6.2$ & $<0.001$ \\
Change & $50.9 \pm 18.4$ & $44.9 \pm 10.6$ & $<0.001$ \\
Function score & & & \\
Preoperative & $43.6 \pm 13.5$ & $40.2 \pm 13.6$ & 0.002 \\
Postoperative & $80.9 \pm 12.4$ & $78.7 \pm 10.9$ & 0.427 \\
Change & $37.3 \pm 15.4$ & $38.5 \pm 15.3$ & 0.201 \\
Range of motion $\left(^{\circ}\right)$ & & & \\
Preoperative & $119.8 \pm 25.7$ & $120.2 \pm 20.7$ & 0.834 \\
Postoperative & $131.4 \pm 10.1$ & $129.0 \pm 12.2$ & 0.008 \\
Change & $11.6 \pm 24.2$ & $8.8 \pm 19.5$ & 0.113 \\
\hline
\end{tabular}

Values are presented as mean \pm standard deviation.

${ }^{a)}$ Group A patients who received the Attune prosthesis.

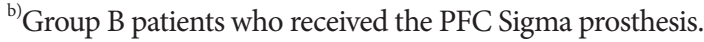

\section{Results}

The KS and FS improved significantly in both groups after TKA $(\mathrm{p}<0.001)$ (Table 2). The postoperative KS was higher in group A (93.1 vs. 88.8, $\mathrm{p}<0.001$ ). The postoperative ROM of group $\mathrm{A}$ was also greater than that of group B $\left(131.4^{\circ}\right.$ vs. $\left.129.0^{\circ}, \mathrm{p}=0.008\right)$.

Radiographically, there were no significant differences in the pre- and postoperative mechanical axis between group $\mathrm{A}$ and group B (Table 3 ). The mean $\alpha, \beta, \gamma$, and $\delta$ angles did not differ significantly between the two groups (Table 3 ).

There were no significant differences in pre- and postoperative patellar thickness between two groups (Table 3). The changes in patella thickness also did not differ significantly between group A and group B (0.7 mm vs. $0.6 \mathrm{~mm}, \mathrm{p}=0.799)$. The residual patella was thinner in group A than group B (14.8 mm vs. $15.7 \mathrm{~mm}$, $\mathrm{p}<0.001$ ) (Table 3). Group A had more patients at high-risk for patellar fractures (7.5\% vs. $2.1 \%, \mathrm{p}=0.003$ ) (Table 4 ).

Regarding the actual occurrence of complications, there was no knee in which a periprosthetic fracture or loosening around the patellar component occurred.

\section{Discussion}

The most important finding of the present study is that the risk of injury to residual patella after TKA might be higher when using Attune, although the prosthesis provided more favorable clinical results than PFC Sigma.

Several previous studies compared the clinical results between
Table 3. Comparison of the Radiographic Results between the Groups

\begin{tabular}{|c|c|c|c|}
\hline Variable & 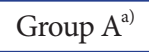 & Group B ${ }^{\text {b) }}$ & $\mathrm{p}$-value \\
\hline \multicolumn{4}{|l|}{ Mechanical axis $\left({ }^{\circ}\right)$} \\
\hline Preoperative & $-11.6 \pm 6.6$ & $-11.7 \pm 7.5$ & 0.751 \\
\hline Postoperative & $-0.9 \pm 2.6$ & $-1.2 \pm 2.8$ & 0.101 \\
\hline Change & $10.7 \pm 6.6$ & $10.5 \pm 7.1$ & 0.145 \\
\hline \multicolumn{4}{|l|}{ Position of components $\left(^{\circ}\right)$} \\
\hline$\alpha$ angle & $95.4 \pm 1.7$ & $95.0 \pm 1.6$ & 0.102 \\
\hline$\beta$ angle & $90.8 \pm 2.1$ & $90.6 \pm 2.2$ & 0.158 \\
\hline$\gamma$ angle & $2.3 \pm 2.9$ & $1.5 \pm 2.3$ & 0.091 \\
\hline$\delta$ angle & $88.7 \pm 2.5$ & $88.5 \pm 2.1$ & 0.101 \\
\hline \multicolumn{4}{|l|}{ Patellar thickness (mm) } \\
\hline Preoperative & $23.1 \pm 3.0$ & $23.6 \pm 3.0$ & 0.088 \\
\hline Postoperative & $23.9 \pm 2.3$ & $24.2 \pm 2.5$ & 0.073 \\
\hline Change & $0.7 \pm 2.9$ & $0.6 \pm 3.4$ & 0.799 \\
\hline $\begin{array}{l}\text { Thickness of the residual } \\
\text { patella }(\mathrm{mm})\end{array}$ & $14.8 \pm 2.1$ & $15.7 \pm 2.4$ & $<0.001$ \\
\hline
\end{tabular}

Values are presented as mean \pm standard deviation.

${ }^{a)}$ Group A patients who received the Attune prosthesis.

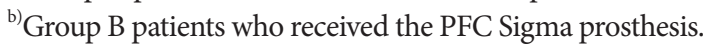

Table 4. Comparison of the Distribution of Residual Patellar Thickness

\begin{tabular}{cccccc}
\hline \multirow{2}{*}{$\begin{array}{c}\text { Thickness } \\
(\mathrm{mm})^{\mathrm{a})}\end{array}$} & \multicolumn{2}{c}{ Group A $^{\mathrm{b})}$} & & \multicolumn{2}{c}{ Group B $^{\mathrm{c})}$} \\
\cline { 2 - 5 } \cline { 5 - 6 } & subjects & $\begin{array}{c}\text { Cumulative } \\
(\%)^{\mathrm{d})}\end{array}$ & & $\begin{array}{c}\text { No. of } \\
\text { subjects }\end{array}$ & $\begin{array}{c}\text { Cumulative } \\
(\%)^{\mathrm{d})}\end{array}$ \\
\hline$<12^{\mathrm{d})}$ & 23 & 7.5 & & 6 & 2.1 \\
$12-14$ & 88 & 36.9 & & 66 & 23.9 \\
$14-16$ & 115 & 75.3 & & 122 & 64.8 \\
$16-18$ & 52 & 92.5 & & 57 & 83.8 \\
$18-20$ & 16 & 97.9 & & 36 & 95.8 \\
$20-22$ & 6 & 100 & & 9 & 98.6 \\
$24-26$ & 0 & 100 & & 2 & 99.3 \\
$26-28$ & 0 & 100 & 0 & 99.3 \\
$28-30$ & 0 & 100 & 2 & 100 \\
\hline
\end{tabular}

a) Thickness of the residual patella.

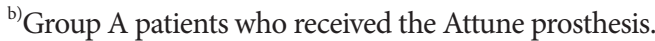

${ }^{c}$ Group B patients who received the PFC Sigma prosthesis.

d) The proportion of high-risk cases with a residual patellar thickness of less than $12 \mathrm{~mm}$ was significantly different between the groups $(\mathrm{p}=0.003)$.

Attune and PFC sigma TKAs ${ }^{8-10)}$. Ranawat et al. ${ }^{8)}$ reported 2-year follow-up clinical results based on Knee Society scores in groups with the above two prostheses: there were no significant differences between the two groups in the postoperative KS (92.4 vs. 92.8, $\mathrm{p}=0.75)$ and FS (89.3 vs. 89.4, $\mathrm{p}=0.096)$, and no difference 
was also shown in the postoperative $\operatorname{ROM}\left(117.0^{\circ}\right.$ vs. $114.2^{\circ}$, $\mathrm{p}=0.025)$. However, in our study, there were better clinical results in the group with Attune with respect to the postoperative KS and ROM. These better results in group A might be associated with improvement in the design that allows for gradual reduction of the femoral radius during knee flexion from $5^{\circ}$ to $70^{\circ}$ and optimization of patellofemoral conformity ${ }^{8-10)}$.

Many previous studies reported that the risk of periprosthetic patellar fracture might increase when the residual patella is too thin $^{4-6)}$. Reuben et al. ${ }^{6)}$ evaluated the strain on the patellofemoral joint in a cadaveric study and concluded that patellar strain was increased when the thickness of the residual patella was less than $15 \mathrm{~mm}$. A clinical study by Seo et al. ${ }^{19)}$ evaluated the various risk factors that might be associated with patellar fractures after TKA. In the review of consecutive 7,866 TKAs, they found that patients with a patellar thickness of less than $12 \mathrm{~mm}$ had a greater risk of patellar fractures (odds ratio, 1.6; $\mathrm{p}<0.043$ ). Although, some literatures had conflicting views on the role of the residual patellar thickness ${ }^{24)}$, basic science and clinical studies supported the theory that a decreased residual patellar thickness would be associated with an increased risk of periprosthetic patellar fractures.

It is known that the postoperative thickness of the prosthesispatellar component should be close to the thickness of the original patella ${ }^{25,26)}$. The patellar components in the Attune were thicker (range, 0.5 to $1.5 \mathrm{~mm}$ ) than those in the PFC Sigma for the generally used size range of $29-38 \mathrm{~mm}$ in Asian patients. The thickness of the residual patella may have been shallow in group A because more of the patellar bone had to be resected, particularly in patients with a small patella ${ }^{4,6)}$. In the present study, the residual patella was thinner in group A than in group B (14.8 mm vs. $15.7 \mathrm{~mm}, \mathrm{p}=0.003$ ); group A had a higher proportion of patients at high-risk for periprosthetic patellar injury with a residual thickness of $<12 \mathrm{~mm}$ (7.5\% vs. $2.1 \%, \mathrm{p}=0.003$ ).

The risk of patellar fractures can be linked to the bony geometric characteristics of Asian people. The patella tends to be thinner in Asians. Kim et al. ${ }^{12)}$ reported that the average thickness of the patella in Koreans is $21.2 \mathrm{~mm}$ in females and $23.1 \mathrm{~mm}$ in males, whereas the corresponding mean thickness in Caucasians is 21.8 to $22.5 \mathrm{~mm}$ in females and 23.9 to $26.1 \mathrm{~mm}$ in males. Therefore, greater precaution will be necessary to avoid patellar fractures in Asian patients, especially when the Attune prosthesis is implanted in small knees with a preoperative patellar thickness of $<20 \mathrm{~mm}$.

After recognizing the risk of patellar injury in the Attune prosthesis, the residual patellar thickness has been advised to be at least $12 \mathrm{~mm}$ when using the latest prosthesis, even in the situation where the postoperative patella is expected to be thicker than the preoperative patella. The patella has been rarely overstuffed because the anterior flange of the current prosthesis is shallower than that of the previous one ${ }^{10)}$. If patellar overstuffing is expected, it could be prevented by displacing the femoral component posteriorly to the extent that notching of the anterior femoral cortex or excessive narrowing of the flexion gap does not occur.

This study has several limitations attributable to the retrospective design and relatively short follow-up duration. First, we assessed the injury risk based on radiographic measurements instead of the evaluation of actual complications after a long-term followup. The average 2-year follow-up could be sufficient for evaluation of radiographic parameters for the patellar thickness, not for the investigation of the occurrence of postoperative complications. Furthermore, regarding the low incidence rate of patellar fractures after TKA (about 1\%), the number of cases in each group does not seem to be sufficient for proper intergroup comparison. Therefore, a larger cohort study with a longer follow up will be required. Second, cartilage thickness was not taken into consideration in the evaluation of the patellar thickness. This is the reason why the change of patellar thickness had a positive value. However, we followed the surgical principle that the original patellar thickness should be preserved or slightly decreased by $0.5 \mathrm{~mm}$. In addition, measurement of the cartilage thickness was not considered necessary because the critical parameter of the present study was the residual patellar thickness. Third, most of the patients in the present study were Korean females with small knees, which should be considered when extrapolating our findings to other populations. However, based on our findings, we would like to emphasize the need for caution when using the Attune prosthesis in Asian patients to avoid the risk of injury to the residual patella.

\section{Conclusions}

The Attune knee system provided slightly better clinical results than the PFC Sigma prosthesis in TKA. However, the injury risk to the residual patella was increased with use of the Attune prosthesis in Asian patients.

\section{Conflict of Interest}

No potential conflict of interest relevant to this article was reported.

\section{References}

1. Sheth NP, Pedowitz DI, Lonner JH. Periprosthetic patellar 
fractures. J Bone Joint Surg Am. 2007;89:2285-96.

2. Konan S, Sandiford N, Unno F, Masri BS, Garbuz DS, Duncan CP. Periprosthetic fractures associated with total knee arthroplasty: an update. Bone Joint J. 2016;98:1489-96.

3. Yoo JD, Kim NK. Periprosthetic fractures following total knee arthroplasty. Knee Surg Relat Res. 2015;27:1-9.

4. Amirouche F, Choi KW, Goldstein WM, Gonzalez MH, Broviak S. Finite element analysis of resurfacing depth and obliquity on patella stress and stability in TKA. J Arthroplasty. 2013;28:978-84.

5. Oishi CS, Kaufman KR, Irby SE, Colwell CW Jr. Effects of patellar thickness on compression and shear forces in total knee arthroplasty. Clin Orthop Relat Res. 1996;(331):283-90.

6. Reuben JD, McDonald CL, Woodard PL, Hennington LJ. Effect of patella thickness on patella strain following total knee arthroplasty. J Arthroplasty. 1991;6:251-8.

7. DePuy Synthes. ATTUNE ${ }^{\oplus}$ knee system [Internet]. Warsaw, IN: DePuy Synthes; 2018 [cited 2017 Sep 8]. Available from: https://www.depuysynthes.com/hcp/knee/products/qs/ ATTUNE-Knee-System.

8. Ranawat CS, White PB, West S, Ranawat AS. Clinical and radiographic results of attune and PFC sigma knee designs at 2-year follow-up: a prospective matched-pair analysis. J Arthroplasty. 2017;32:431-6.

9. Webb JE, Yang HY, Collins JE, Losina E, Thornhill TS, Katz JN. The evolution of implant design decreases the incidence of lateral release in primary total knee arthroplasty. J Arthroplasty. 2017;32:1505-9.

10. Martin JR, Jennings JM, Watters TS, Levy DL, McNabb DC, Dennis DA. Femoral implant design modification decreases the incidence of patellar crepitus in total knee arthroplasty. J Arthroplasty. 2017;32:1310-3.

11. DePuy Synthes. SIGMA ${ }^{\star}$ total knee system [Internet]. Warsaw, IN: DePuy Synthes; 2018 [cited 2017 Sep 8]. Available from: https://www.depuysynthes.com/hcp/knee/products/ qs/SIGMA-Total-Knee-System.

12. Kim TK, Chung BJ, Kang YG, Chang CB, Seong SC. Clinical implications of anthropometric patellar dimensions for TKA in Asians. Clin Orthop Relat Res. 2009;467:1007-14.

13. Pierce TP, Jauregui JJ, Cherian JJ, Elmallah RK, Harwin SF, Mont MA. Is there an ideal patellar thickness following total knee arthroplasty? Orthopedics. 2016;39:e187-92.

14. Bae DK, Baek JH, Yoon KT, Son HS, Song SJ. Comparison of patellofemoral outcomes after TKA using two prostheses with different patellofemoral design features. Knee Surg
Sports Traumatol Arthrosc. 2017;25:3747-54.

15. Insall JN, Dorr LD, Scott RD, Scott WN. Rationale of the Knee Society clinical rating system. Clin Orthop Relat Res. 1989;(248):13-4.

16. Ewald FC. The Knee Society total knee arthroplasty roentgenographic evaluation and scoring system. Clin Orthop Relat Res. 1989;(248):9-12.

17. Kawano T, Miura H, Nagamine R, Urabe K, Matsuda S, Mawatari T, Moro-Oka T, Iwamoto Y. Factors affecting patellar tracking after total knee arthroplasty. J Arthroplasty. 2002;17:942-7.

18. Lie DT, Gloria N, Amis AA, Lee BP, Yeo SJ, Chou SM. Patellar resection during total knee arthroplasty: effect on bone strain and fracture risk. Knee Surg Sports Traumatol Arthrosc. 2005;13:203-8.

19. Seo JG, Moon YW, Park SH, Lee JH, Kang HM, Kim SM. A case-control study of spontaneous patellar fractures following primary total knee replacement. J Bone Joint Surg Br. 2012;94:908-13.

20. Stickley CD, Wages JJ, Hetzler RK, Andrews SN, Nakasone CK. Standard radiographs are not sufficient for assessing knee mechanical axis in patients with advanced osteoarthritis. J Arthroplasty. 2017;32:1013-7.

21. Ewald FC. The Knee Society total knee arthroplasty roentgenographic evaluation and scoring system. Clin Orthop Relat Res. 1989;(248):9-12.

22. Bae DK, Song SJ, Kim HJ, Seo JW. Change in limb length after high tibial osteotomy using computer-assisted surgery: a comparative study of closed- and open-wedge osteotomies. Knee Surg Sports Traumatol Arthrosc. 2013;21:120-6.

23. Cabuk H, İmren Y, Tekin AC, Dedeoglu SS, Gurbuz H. High varus angle and lower posterior tibial slope associated with PCL injury in cruciate retaining total knee arthroplasty: an MRI study. J Knee Surg. 2018;31:277-83.

24. Ritter MA, Pierce MJ, Zhou H, Meding JB, Faris PM, Keating EM. Patellar complications (total knee arthroplasty): effect of lateral release and thickness. Clin Orthop Relat Res. 1999;(367):149-57.

25. Baldwin JL, House CK. Anatomic dimensions of the patella measured during total knee arthroplasty. J Arthroplasty. 2005;20:250-7.

26. Hsu HC, Luo ZP, Rand JA, An KN. Influence of patellar thickness on patellar tracking and patellofemoral contact characteristics after total knee arthroplasty. J Arthroplasty. 1996;11:69-80. 